\title{
Percepción, disposición y compromiso docente de las prácticas de gestión educativa en universidades mexicanas
}

\author{
Armando Sánchez Macías ${ }^{1}$ \\ José Zilberstein Toruncha ${ }^{2}$ \\ Isabel Cristina Flores Rueda ${ }^{3}$ \\ Virginia Azuara Pugliese 4
}

\begin{abstract}
Resumen
El ser humano, el contexto donde se desenvuelve y sus aspiraciones son cambiantes por lo que las instituciones educativas están obligadas a ser dinámicas y flexibles (Nava, Glasserman y Torres, 2019). El objetivo del presente estudio es conocer la posible relación entre las directrices esenciales de la gestión educativa: la disposición, el compromiso y la percepción de los docentes. El trabajo resume los resultados de una investigación de tipo cuantitativa con enfoque mixto, descriptivacorrelacional, transversal y no experimental, que se apoyó en un cuestionario el cual incluye tres dimensiones consideradas en la gestión educativa: gestión institucional, escolar y pedagógica. Para lo cual, se estudiaron y compararon opiniones de 93 docentes de instituciones públicas y privadas en San Luis Potosí, México. Los principales resultados indican que el tipo de financiamiento, es el principal factor que determina los niveles de gestión en las IES, siendo el de tipo privado el que alcanza mejores niveles que el público. Así mismo que el último nivel académico y la formación pedagógica formal no tiene una influencia
\end{abstract}

\footnotetext{
${ }^{1}$ Doctor en Educación. Profesor Investigador de la Coordinación Académica Región Altiplano Oeste. Universidad Autónoma de San Luis Potosí, México. https://orcid.org/0000-0001-9575-3248. armando.sanchez@uaslp.mx.

2 Doctor en Ciencias Pedagógicas. Director del Campus Tequis de la Universidad Tangamanga, México. https://orcid.org/0000-0002-5893-5090.jzilberstein@utan.edu.mx.

3 Maestra em Educación. Profesora Investigadora de la la Coordinación Académica Región Altiplano Oeste. Universidade Autônoma de San Luis Potosí, México. http://orcid.org/0000-0003-1257-2893. isabel.flores@uaslp.mx.

${ }^{4}$ Doctora en Mercadotecnia. Coordinadora de la Licenciatura en Administración en la Coordinación Académica Región Altiplano Oeste. Universidad Autónoma de San Luis Potosí, México. https://orcid.org/0000-0001-83909188.virginia.azuara@uaslp.mx.
} 
en los niveles de gestión educativa. Finalmente la gestión pedagógica muestra diferencias en sus resultados estadísticamente significativas con un mejor valor en las privadas que en las públicas, siendo la práctica constructivista su indicador clave.

Palabras clave: Gestión educativa. Administración educativa. Gestión de instituciones de educación superior.

Percepção, disposição e compromisso docente das práticas de gestão educacional nas universidades mexicanas

\section{Resumo}

O ser humano, o contexto em que atua e suas aspirações estão mudando, de modo que as instituições educacionais são forçadas a serem dinâmicas e flexíveis (Nava, Glasserman e Torres, 2019). O objetivo deste estudo é compreender a possível relação entre as diretrizes essenciais da gestão educacional: a disposição, o comprometimento e a percepção dos professores. O trabalho resume os resultados de uma investigação quantitativa com uma abordagem mista, descritivo-correlacional, transversal e não experimental, respaldada por um questionário que inclui três dimensões consideradas na gestão educacional: gestão institucional, escolar e pedagógica. Para isso, foram estudadas e comparadas as opiniões de 93 professores de instituições públicas e privadas de San Luis Potosí, México. Os principais resultados indicam que o tipo de financiamento é o principal fator que determina os níveis de gestão nas IES, e o tipo privado atinge níveis mais altos que o público. Da mesma forma, o último nível acadêmico e a formação pedagógica formal não influenciam os níveis de gestão educacional. Por fim, a gestão pedagógica mostra diferenças estatisticamente significantes em seus resultados, com melhor valor no privado do que no público, sendo a prática construtivista o principal indicador.

Palavras-chave: Gestão educacional. Administração educacional. Gestão de instituições de ensino superior.

Perception, disposition and teaching commitment of educational management practices in Mexican universities 


\begin{abstract}
The human being, the context in which he operates and his aspirations are changing, so educational institutions are obliged to be dynamic and flexible (Nava, Glasserman and Torres, 2019). The objective of this study is to know the possible relationship between the essential guidelines of educational management: the willingness, commitment and perception of teachers. The paper summarizes the results of a quantitative investigation with a mixed, descriptive-correlational, transversal and non-experimental approach, which was supported by a questionnaire that includes three dimensions considered in educational management: institutional, school and pedagogical management. For this, the opinions of 93 teachers from public and private institutions in San Luis Potosí, Mexico were studied and compared. The main results indicate that the type of financing is the main factor that determines the levels of management in HEIs, and the private type reaches higher levels than the public. Likewise, the last academic level and formal pedagogical training do not influence levels of educational management. Finally, pedagogical management shows statistically significant differences in its results with a better value in private than in public, with constructivist practice being its key indicator.
\end{abstract}

Key words: Educational management. Educational administration. Management of higher education institutions.

\title{
$* * *$
}

\section{Introducción}

Las Instituciones de Educación Superior (IES) como cualquier otro tipo de organizaciones están formadas por talentos humanos apoyados en recursos materiales, que persiguen un objetivo en común y que para ello acuerdan ejes y vías para alcanzarlos. Existe presente la pretensión de utilizar la menor cantidad de recursos para este propósito, es decir ser eficiente, a la vez, que se debe tener un enfoque de logro, es decir eficacia, porque de nada sirve optimizar recursos si no se alcanzan los objetivos. En otras palabras: "[...] la eficiencia se concentra en cómo se hacen las cosas, de qué modo se ejecutan. La eficacia en: para qué se hacen las cosas, cuáles son 
los resultados que se persiguen, qué objetivos se logran" (SÁNCHEZ MACÍAS, 2016, pág. 36).

Entre los recursos que deben ser movilizados de manera coordinada hacia el logro, se encuentran las voluntades humanas, con todas sus complejidades, matices y permanentes variaciones. Barbón Pérez y Fernández Pino (2016) consideran que la gestión educativa parte del Enfoque Procesal (FAYOL, 1949): planeación, organización, regulación y control. Asimismo, afirman que deben acompañar el despliegue de las actividades sustantivas de las IES. Lemos (2017), reconoce que en países como Brasil los pioneros de la gestión educativa asumieron como fundamento las teorías científica (Taylor) y clásica de la Administración (Fayol). Otras posturas como la de Oplatka (2019), apuntan a la creación de una gestión educativa Latinoamericana que no se adscriba a conceptos importados.

Los autores de este trabajo asumen que no existe una mejor manera para lograr los propósitos de la organización educativa que la aplicación de las Ciencias Administrativas, las cuales, a su vez, basado en el Enfoque Procesal de Fayol (1949) incluye dos grandes momentos, la Administración y la Gestión.

El primer momento se refiere al aspecto estático que incluye las fases de planeación y organización ¿qué, para qué y cómo se va a hacer?

El segundo momento se centra en el elemento dinámico que incluye la dirección y la gestión, es decir hacer que las cosas sucedan. Dicho enfoque de gestión en la últimos años, ha dado pie a diversas políticas públicas, investigaciones y experiencias, en relación con “[...] el mejoramiento de la calidad educativa, contribuyendo a enseñar a las escuelas a que puedan tomar decisiones claves" (BALDERRAMA, 2006, pág. 11).

Cuando la administración y la gestión se refieren al campo educativo son conocidas como administración y gestión educativas. Una de las visiones en este sentido es que la administración educativa es una disciplina incluida en las Ciencias Pedagógicas y se encarga del estudio de la organización 
educativa (HERNÁNDEZ ROJAS, 1998). Esta, a su vez, está integrada por: (a) el proyecto educativo; (b) el paradigma educativo puesto en práctica; (c) la infraestructura general; (d) el educador; (e) el educando; (f) la familia y; (g) otros actores sociales. Otro enfoque considera que la administración y gestión educativas son la aplicación de los principios de las Ciencias Administrativas en el campo de las organizaciones que tienen por objeto la educación (las escuelas). Para Casassus (2002) la gestión educativa es una disciplina de alto grado de flexibilidad debido a los recurrentes cambios en los que está inmersa, es decir, busca aplicar los argumentos de la gestión en el campo educativo, en este sentido, es una disciplina aplicada al campo de acción. Connolly, James y Fertig (2019) la asumen como llevar la responsabilidad del adecuado funicionamiento del sistema en una institución educativa en la que intervienen varias personas. Por su parte Sánchez Macías (2013), considera que:

[...] la administración educativa no es una ciencia como tal, sino la aplicación de la ciencia administrativa (con sus principios) al campo específico de las instituciones, sistemas u organismos que conciben, modelan, coordinan y dirigen los procesos educativos. Tiene un carácter mayormente conceptual y ligeramente operativo. Por su parte, la Gestión educativa corresponde a la práctica, operación y ejecución de los procesos educativos en sus distintas dimensiones: institucional, escolar y pedagógica. Implica mayormente el despliegue de habilidades, destrezas y técnicas operativas aplicadas con maestría y pericia para conseguir que las cosas se hagan (p.11).

La Secretaría de Educación Pública (SEP, 2009) - autoridad educativa en México - asume la siguiente postura al respecto. La administración educativa se concibe como gestión educativa, bajo un modelo multidimensional, en el cual, cada dimensión contiene al siguiente y así sucesivamente, a saber: (a) la gestión educativa se refiere al sistema 
educativo en general; (b) gestión institucional, acciones referidas a la estructura organizacional con acciones de orden administrativo, gerencial, financiero y de personal; (c) gestión escolar, es la que se orienta a generar las condiciones apropiadas en la comunidad educativa: alumnos, directivos, administrativos y padres de familia, para lograr los propósitos educativos y finalmente; (d) la gestión pedagógica, es decir lo que ocurre en el aula, en la cual, el docente hace un despliegue de estrategias didácticas y pedagógicas para lograr los objetivos y/o competencias que se establecen en los planes y programas de estudio.

En las últimas dos décadas la unión de la gestión y la educación constituyen una relación con raíces económicas, es decir, para la gestión educativa se comprenden conceptos como evaluación, competitividad, incentivos, eficiencia y eficacia. Los conceptos anteriores pueden contraponerse con el universo docente concentrado de corrientes sociológicas, psicológicas y de pedagogía. Por lo anterior, es de alta relevancia conocer si ambos universos no mantienen un doble lenguaje entre directivos (planificadores estratégicos) y docentes (encargados de la ejecución) quiénes pueden confundirse con las expresiones de rendimiento institucional (CASASSUS, 2002). Trabajos como el de Aravena Castillo y Hallinger (2018) muestran que la investigación en el campo de la Gestión y Liderazgo Educativo en Latinoamérica se concentran en los últimos diez años; mientras que Bertel-Narváez, Viloria-Escobar y Sánchez-Buitrago (2019) encontraron que los temas abordados principalmente son la gestión académica, gestión comunitaria y directiva, así como en gestión administrativa y financiera, en ese orden. Franco Garcia (2018) considera que se gesta una gestión educativa mexicana a partir de las crisis y transformaciones sufridas en los ochentas, el aporte más significativo es la intergración de las colectivos escolares en la toma de decisiones.

Los autores de este artículo, asumen los niveles establecidos por la SEP (2009) desde y hacia los cuales desplegar las acciones para el logro de los objetivos educativos con eficacia y eficiencia (SÁNCHEZ MACÍAS, 2016), 
así como según el Enfoque Basado en Competencias (ZILBERSTEIN, 2015) y bajo una visión de administración y gestión educativa (Sánchez Macías, 2013) de las IES se considera relevante identificar ¿cuáles deben ser dichos objetivos en el momento histórico en que se encuentran las IES y bajo el contexto político, social y económico que priva en un país como México?

Sánchez Macías y Zilberstein Toruncha (2019) proponen orientar dichos esfuerzos para atender once retos que a juicio de los autores permiten desarrollar al máximo las potencialidades de la comunidad educativa, “[...] teniendo en consideración al mundo empresarial pero no orientado a sus intereses particulares sino a los generales" (p.3). Los retos propuestos son los siguientes (SÁNCHEZ MACÍAS; ZILBERSTEIN TORUNCHA, 2019): (a) acceso universal y de calidad a la educación para todos los estudiantes universitarios; (b) reducción de la deserción universitaria, con énfasis en el primer año de las carreras; (c) centrar la educación universitaria en la preparación para el ejercicio de la profesión y el compromiso con el servicio a la sociedad; (d) promover la generación de nuevos conocimientos, que traiga por consecuencia la innovación y con ello la mentalidad de estudiantes innovadores; (e) diseño de actividades que propicien el desarrollo en el contexto real de la ciencia y la tecnología; (f) aumentar como resultado de la investigación de estudiantes y docentes universitarios, la producción científica y con ello las patentes y registros de propiedad de mexicanas y mexicanos; (g) enfoque en la práctica pedagógica hacia la educación y no limitado a la instrucción o enseñanza; (h) formación ética, de servicio en la profesión de los estudiantes de las universidades; (i) actuación integrada al contexto, para lo cual es imprescindible la formación y rápida inserción laboral de sus egresados, trabajar en redes de intercambio e involucrar a sus docentes y estudiantes en las mismas; (j) impulsar el dominio generalizado en los estudiantes universitarios de la tecnología, y sus aplicaciones concretas en el área del conocimiento que estudian, de manera tal que al culminar sus estudios puedan aplicarlo en el ejercicio de la profesión y; (k) 
diseñar procesos de autoevaluación de las universidades que creen una cultura de evaluación, que se vincule con las evaluaciones externas.

Dichos retos visualizados desde una perspectiva administrativa y de gestión se pueden agrupar dentro de los niveles de actuación de la gestión educativa (institucional, escolar y pedagógica) asumidos por la SEP (2009) desarrollando una definición operacional con dimensiones e indicadores, los cuales se presenta en la Figura 1.

Figura 1: Definición operacional de la Gestión educativa

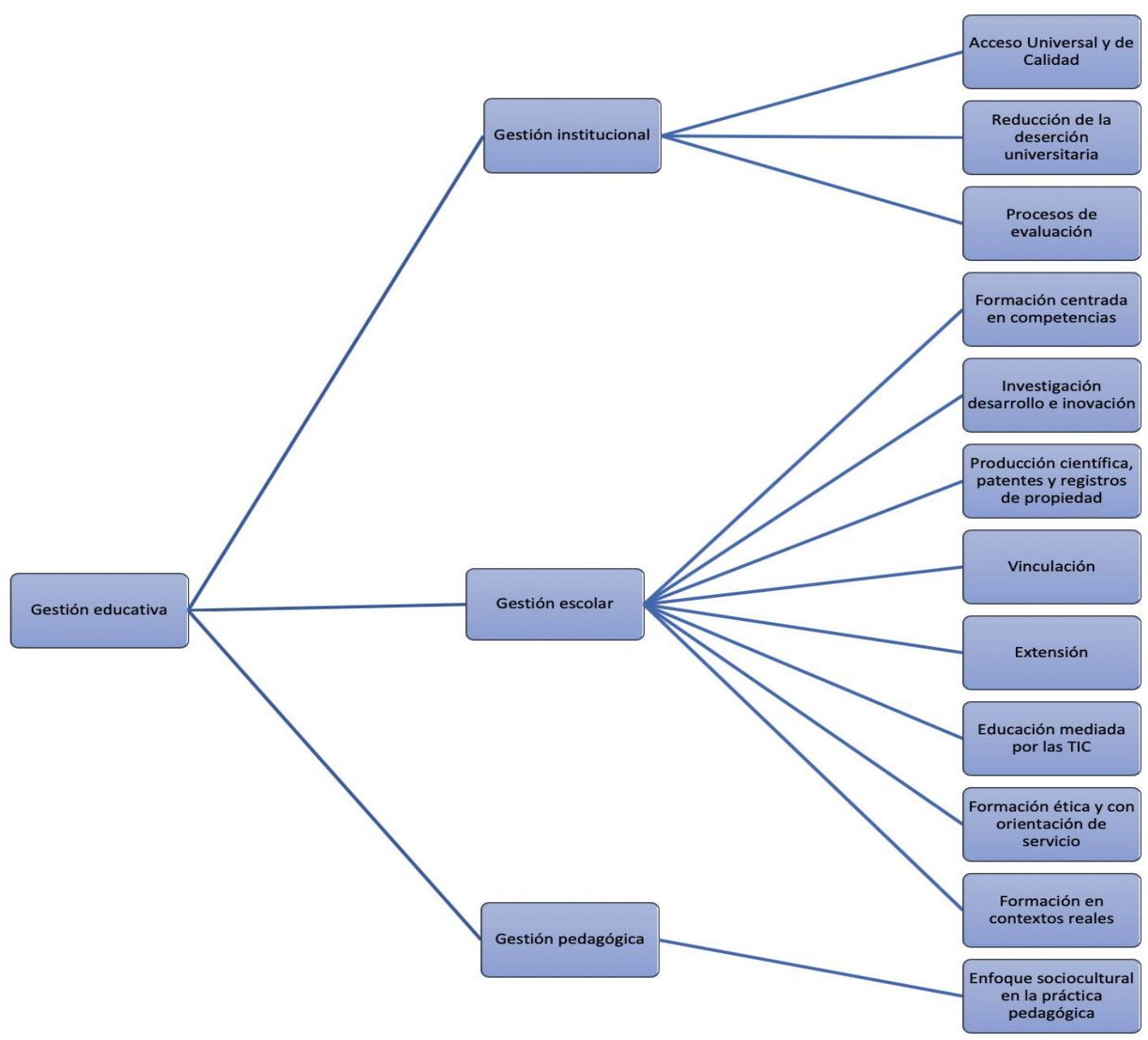

Fuente: Adaptado de Pigozzi, 2008; Medina Palomera, Amado Moreno y Brito Páez, 2010; Valera Sierra, 2010; SEMS, 2014; García Galván, 2017; Di Bello y Romero, 2018; Zilberstein Toruncha y Olmedo Cruz, 2018; Sánchez Macías, Zilberstein Toruncha y Azuara Pugliese, 2018; Sánchez Macías y Veytia Bucheli, 2019. 
Dentro de la formación por competencias se asumen posturas del Enfoque Histórico Cultural (Zilberstein, Silvestre, Olmedo, 2016), atendiendo a los tipos de actividad que debe realizar un estudiante universitario: cognoscitiva, práctica, valorativa y comunicativa.

\section{Diseño metodológico}

En este contexto, el objetivo del presente texto es conocer la posible relación entre elementos que conforman las directrices esenciales de la gestión educativa y la disposición, el compromiso y la percepción de los docentes tanto en instituciones privadas como públicas del nivel educativo superior en México.

Es una investigación de tipo cuantitativa con enfoque mixto, descriptiva-correlacional, transversal y no experimental. Basada en la definición operacional (Figura 1) se desarrolla un cuestionario que incluye las tres dimensiones de la gestión educativa (institucional, escolar y pedagógica), 14 indicadores y 36 ítems en total. El cuestionario utiliza una escala Likert de cinco niveles y tres momentos de respuesta a saber: (a) identificar ¿qué tan de acuerdo está el docente con la realización de las acciones medidas?; (b) en segundo lugar, ¿qué tan dispuesto y comprometido se muestra para involucrarse en su puesta en marcha? y; (c) en tercer lugar ¿cuál es la percepción que tiene acerca del nivel de implementación de estas acciones en la IES donde colabora?

La población fueron profesores de IES de la Ciudad de San Luis Potosí. La muestra estuvo integrada por 93 docentes de nivel superior de instituciones públicas y privadas. El muestreo fue no probabilístico por conveniencia. La medición de la confiabilidad del instrumento se llevó a cabo mediante el estadístico alpha de Cronbach, los resultados se presentan en las Tablas 1 y 2 . 
Tabla 1: Análisis de la consistencia interna mediante Alpha de Cronbach

\begin{tabular}{|lcccccc|}
\hline & & & \multicolumn{2}{c}{$\begin{array}{c}\text { ¿Qué tan dispuesto } \\
\text { está tan de acuerdo } \\
\text { están las acciones? } \\
\text { comprometerse con } \\
\text { las acciones? }\end{array}$} & $\begin{array}{c}\text { ¿En qué grado percibe } \\
\text { la implementación de } \\
\text { las acciones de parte } \\
\text { de la IES? }\end{array}$ \\
\hline Dimensión & $\begin{array}{c}\text { Alpha de } \\
\text { Cronbach }\end{array}$ & No. de & Alpha de & No. de & Alpha de & No. de \\
Gestión institucional & 0.963 & 14 & 0.971 & 14 & 0.966 & 14 \\
Gestión escolar & 0.987 & 19 & 0.991 & 19 & 0.985 & 19 \\
Gestión pedagógica & 0.931 & 3 & 0.912 & 3 & 0.911 & 3 \\
\hline
\end{tabular}

Fuente: Elaboración propia

Como se observa todas las dimensiones puntuaron por encima del 0.900 lo cual pone de manifiesto la fiabilidad de la escala por la alta consistencia interna del instrumento.

Tabla 2: Análisis de la consistencia interna mediante el análisis de dos mitades

\begin{tabular}{|c|c|c|c|c|c|c|c|c|c|c|c|c|}
\hline \multirow[b]{2}{*}{ Dimensión } & \multicolumn{4}{|c|}{$\begin{array}{c}\text { ¿Qué tan de acuerdo está con } \\
\text { las acciones? }\end{array}$} & \multicolumn{4}{|c|}{$\begin{array}{c}\text { ¿Qué tan dispuesto está a } \\
\text { involucrarse y/o comprometerse } \\
\text { con las acciones? }\end{array}$} & \multicolumn{4}{|c|}{$\begin{array}{c}\text { ¿En qué grado percibe la } \\
\text { implementación de las acciones } \\
\text { de parte de la IES? }\end{array}$} \\
\hline & $\begin{array}{c}\text { Mitad } \\
\quad 1\end{array}$ & $\begin{array}{l}\text { No. } \\
\text { de } \\
\text { items }\end{array}$ & $\begin{array}{l}\text { Mitad } \\
\quad 2\end{array}$ & $\begin{array}{l}\text { No. } \\
\text { de } \\
\text { items }\end{array}$ & $\begin{array}{c}\text { Mitad } \\
\quad 1\end{array}$ & $\begin{array}{l}\text { No. } \\
\text { de } \\
\text { items }\end{array}$ & $\begin{array}{l}\text { Mitad } \\
\quad 2\end{array}$ & $\begin{array}{l}\text { No. } \\
\text { de } \\
\text { items }\end{array}$ & $\begin{array}{c}\text { Mitad } \\
\quad 1\end{array}$ & $\begin{array}{l}\text { No. } \\
\text { de } \\
\text { items }\end{array}$ & $\begin{array}{l}\text { Mitad } \\
\quad 2\end{array}$ & $\begin{array}{c}\text { No. } \\
\text { de } \\
\text { items }\end{array}$ \\
\hline $\begin{array}{l}\text { Gestión } \\
\text { institucional }\end{array}$ & 0.945 & 7 & 0.932 & 7 & 0.944 & 7 & 0.958 & 7 & 0.952 & 7 & 0.929 & 7 \\
\hline $\begin{array}{l}\text { Gestión } \\
\text { escolar }\end{array}$ & 0.977 & 10 & 0.980 & 9 & 0.986 & 10 & 0.979 & 9 & 0.975 & 10 & 0.977 & 9 \\
\hline $\begin{array}{l}\text { Gestión } \\
\text { pedagógica }\end{array}$ & na & & na & & na & & na & & na & & na & \\
\hline
\end{tabular}

Fuente: Elaboración propia

En la Tabla 2 se muestra el análisis de dos mitades en el que de igual manera se encontraron índices superiores a 0.900 , lo cual refuerza la afirmación de la alta consistencia interna y fiabilidad en las dimensiones analizadas. Una vez realizadas las mediciones de confiabilidad de la escala, se procedió a plantear las siguientes hipótesis con respecto la percepción del 
docente con respecto a las tres directrices señaladas; institucional, escolar y pedagógica.

H1 Los niveles de gestión educativa percibida por los docentes están relacionados de manera estadísticamente significativa con el tipo de financiamiento de la IES (público o privado).

H2 Los niveles de gestión educativa percibida por los docentes están relacionados de manera estadísticamente significativa con su último nivel educativo.

H3 Los niveles de gestión educativa percibida por los docentes están relacionados de manera estadísticamente significativa con su formación pedagógica formal.

\section{Resultados}

La muestra seleccionada cuenta con las características sociodemográficas que se muestran en las Tablas 3 y 4.

Tabla 3: Descripción sociodemográfica de la muestra (primera parte)

\begin{tabular}{|c|c|c|c|c|c|c|c|}
\hline \multicolumn{2}{|c|}{$\begin{array}{c}\text { Tipo de } \\
\text { financiamiento de la } \\
\text { IES }\end{array}$} & \multicolumn{2}{|c|}{ Género } & \multicolumn{2}{|c|}{$\begin{array}{l}\text { Último grado de } \\
\text { estudio }\end{array}$} & \multicolumn{2}{|c|}{$\begin{array}{c}\text { Nivel en el que } \\
\text { mayormente imparte } \\
\text { clase }\end{array}$} \\
\hline Público & $36 \%$ & Masculino & $53 \%$ & Licenciatura & $10 \%$ & Licenciatura & $74 \%$ \\
\hline \multirow[t]{3}{*}{ Privado } & $64 \%$ & Femenino & $47 \%$ & Especialidad & $1 \%$ & Posgrado & $26 \%$ \\
\hline & & & & Maestría & $56 \%$ & & \\
\hline & & & & Doctorado & $33 \%$ & & \\
\hline
\end{tabular}

Fuente: Elaboración propia

Acerca de la muestra, el $64 \%$ de docentes son provenientes de escuelas privadas y el resto de públicas, por su parte, el género estuvo equilibrado. Además, cabe destacar que el 90\% de los profesores entrevistados cuentan con alguno posgrado y que la tercera parte inclusive cuentan con doctorado. Estos datos son relevantes debido a que ponen de manifiesto el aumento de grado de habilitación en la planta docente. Esto se refuerza al identificar que casi tres cuartas partes de ellos es docente en 
licenciatura, en la cual, no existe en México, una obligación legal de ostentar un posgrado para desempeñarse, y sin embargo existen los niveles de habilitación que se muestran.

Tabla 4: Descripción sociodemográfica de la muestra (segunda parte)

\begin{tabular}{|c|c|c|c|c|c|c|c|}
\hline \multicolumn{2}{|c|}{ Rango de edad } & \multicolumn{2}{|c|}{$\begin{array}{l}\text { Años de experiencia } \\
\text { docente }\end{array}$} & \multicolumn{2}{|c|}{$\begin{array}{c}\text { Años de experiencia } \\
\text { profesional }\end{array}$} & \multicolumn{2}{|c|}{$\begin{array}{l}\text { Tipo de formación pedagógica } \\
\text { formal }\end{array}$} \\
\hline $\begin{array}{l}\text { De } 25 \text { a } 35 \\
\text { años }\end{array}$ & $18 \%$ & $\begin{array}{l}\text { Menos de } 5 \\
\text { años }\end{array}$ & $17 \%$ & $\begin{array}{l}\text { Menos de } 5 \\
\text { años }\end{array}$ & $8 \%$ & Ninguna & $5 \%$ \\
\hline $\begin{array}{l}\text { De } 36 \text { a } 45 \\
\text { años }\end{array}$ & $30 \%$ & $\begin{array}{l}\text { De } 5 \text { a } 10 \\
\text { años }\end{array}$ & $24 \%$ & $\begin{array}{l}\text { De } 5 \text { a } 10 \\
\text { años }\end{array}$ & $23 \%$ & $\begin{array}{l}\text { Cursos, talleres y/o } \\
\text { diplomados en área } \\
\text { educativa o pedagógica }\end{array}$ & $66 \%$ \\
\hline $\begin{array}{l}\text { De } 46 \text { a } 55 \\
\text { años }\end{array}$ & $31 \%$ & $\begin{array}{l}\text { De } 11 \text { a } 15 \\
\text { años }\end{array}$ & $15 \%$ & $\begin{array}{l}\text { De } 11 \text { a } 15 \\
\text { años }\end{array}$ & $14 \%$ & $\begin{array}{l}\text { Licenciatura en área } \\
\text { educativa o pedagógica }\end{array}$ & $5 \%$ \\
\hline $\begin{array}{l}\text { De } 56 \text { a } 65 \\
\text { años }\end{array}$ & $15 \%$ & $\begin{array}{l}\text { De } 16 \text { a } 20 \\
\text { años }\end{array}$ & $12 \%$ & $\begin{array}{l}\text { De } 16 \text { a } 20 \\
\text { años }\end{array}$ & $15 \%$ & $\begin{array}{l}\text { Posgrado en área } \\
\text { educativa o pedagógica }\end{array}$ & $24 \%$ \\
\hline $\begin{array}{l}\text { Más de } 65 \\
\text { años }\end{array}$ & $5 \%$ & $\begin{array}{l}\text { Más de } 20 \\
\text { años }\end{array}$ & $32 \%$ & $\begin{array}{l}\text { Más de } 20 \\
\text { años }\end{array}$ & $41 \%$ & & \\
\hline
\end{tabular}

Fuente: Elaboración propia

En la Tabla 4 se presenta una caracterización acerca de los rangos de edad de la muestra. Cabe destacar que el 61\% de los profesores se encuentran entre los 36 y 55 años, lo que pone de manifiesto que la planta es mayormente joven. Inclusive, el 18\% no excede los 35 años. En cuanto a la experiencia docente cabe destacar que la gran mayoría (83\%) ha impartido durante cinco años clase frente a grupo y que casi una tercera parte con más de 20 años en la labor. Una situación similar se observa en la experiencia profesional con un 41\% con más de 20 años y una proporción pequeña de $8 \%$ con menos de cinco años. Finalmente se observa que la mayoría, un 95\% ha tenido formación pedagógica formal. Es notable que casi una cuarta parte cuente con un posgrado en el área pedagógica o educativa.

A continuación se presenta el comparativo de resultados por indicador. En la Figura 2 se presentan los resultados de los profesores de las instituciones públicas, en términos generales se observa que el grado de implementación de las acciones por parte de las IES es el que alcanza el nivel más alto, excepto en el indicador de evaluación interna y externa. En segundo lugar se identifica el nivel de acuerdo con las acciones que se 
analizaron, en un nivel muy cercano pero inferior en la mayoría de los indicadores. Esta desviación se puede interpretar como que existe una diferencia -aunque pequeña- entre las acciones que emprenden las IES públicas y lo que los profesores consideran debería de hacerse. En cuanto a la disposición para involucrarse con las acciones se identifica una clara diferencia entre las acciones que se identifican se hacen, el acuerdo con ellas y la voluntad para participar de ellas. Aunque la mayoría de los indicadores se encuentran por encima del 3 (excepto la vinculación) es claro que hay una falta de interés y lo cual puede incluso interpretarse como falta de compromiso hacia los objetivos de la institución. Cabe destacar que el acceso universal, la calidad educativa $\mathrm{y}$ el modelo educativo basado en competencias son los que se destacan por alcanzar niveles más altos. Por su parte la vinculación alcanza niveles inferiores al indiferente.

Figura 2: Resultados por indicadores entidades (IES) públicas

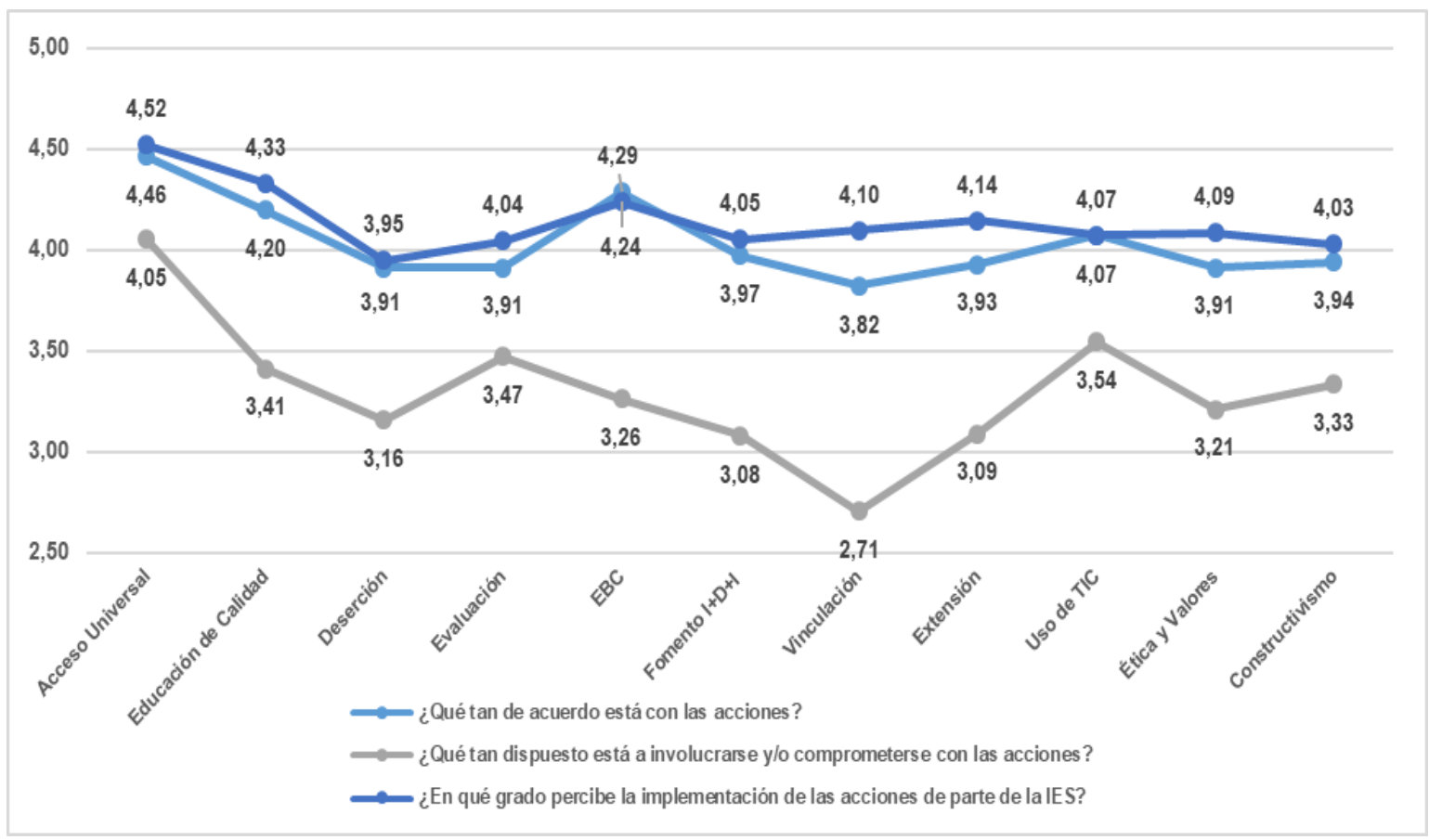

Fuente: Elaboración propia

En la Figura 3 se muestra el análisis realizado en las IES privadas, salta a la vista que los resultados se ordenan de manera inversa, es decir la 
intención de compromiso e involucramiento de parte de los profesores en casi todos los indicadores se encuentra por encima. Le sigue el acuerdo con las acciones y finalmente la percepción de la implementación de estas que hacen las IES privadas. Si bien las gráficas tienen diferente orden, hay que resaltar que los indicadores en las públicas oscilan entre 2.71 y 4.52, mientras que en las privadas entre 3.88 y 4.62, es decir, en promedio en las IES privadas los tres niveles analizados son mas altos. Las acciones percibidas como menos implementadas en las privadas son el fomento $\mathrm{I}+\mathrm{D}+\mathrm{I}$, la vinculación y la extensión, aunque vale aclarar que se encuentran en niveles casi apropiados (nivel 4).

Figura 3: Resultados por indicadores entidades (IES) privadas

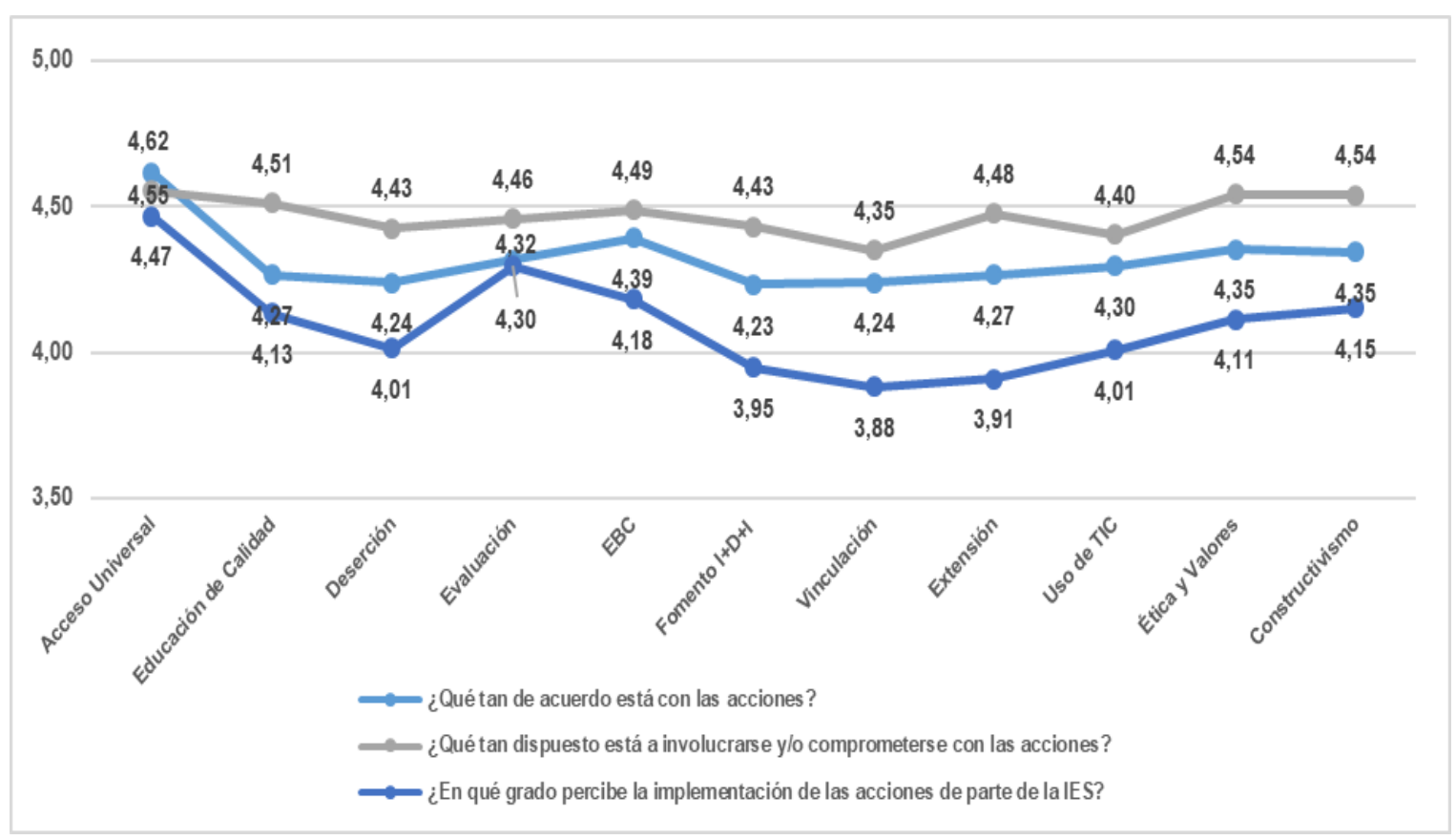

Fuente: Elaboración propia

En la Figura 4 se presenta una comparativa de las medias de los niveles de gestión de acuerdo a las perspectivas analizadas. Como se observa los resultados son más altos en las IES privadas en todos los aspectos. Por otro lado se observa que existe una tendencia inversa entre las IES públicas y las privadas, la cual se hace más patente cuando se refiere al aspecto de 
involucramiento y compromiso, menos marcado en el acuerdo que tienen los profesores con las acciones y casi nulo entre el nivel de implementación.

Figura 4: Promedio de niveles de gestión entre IES públicas y privadas

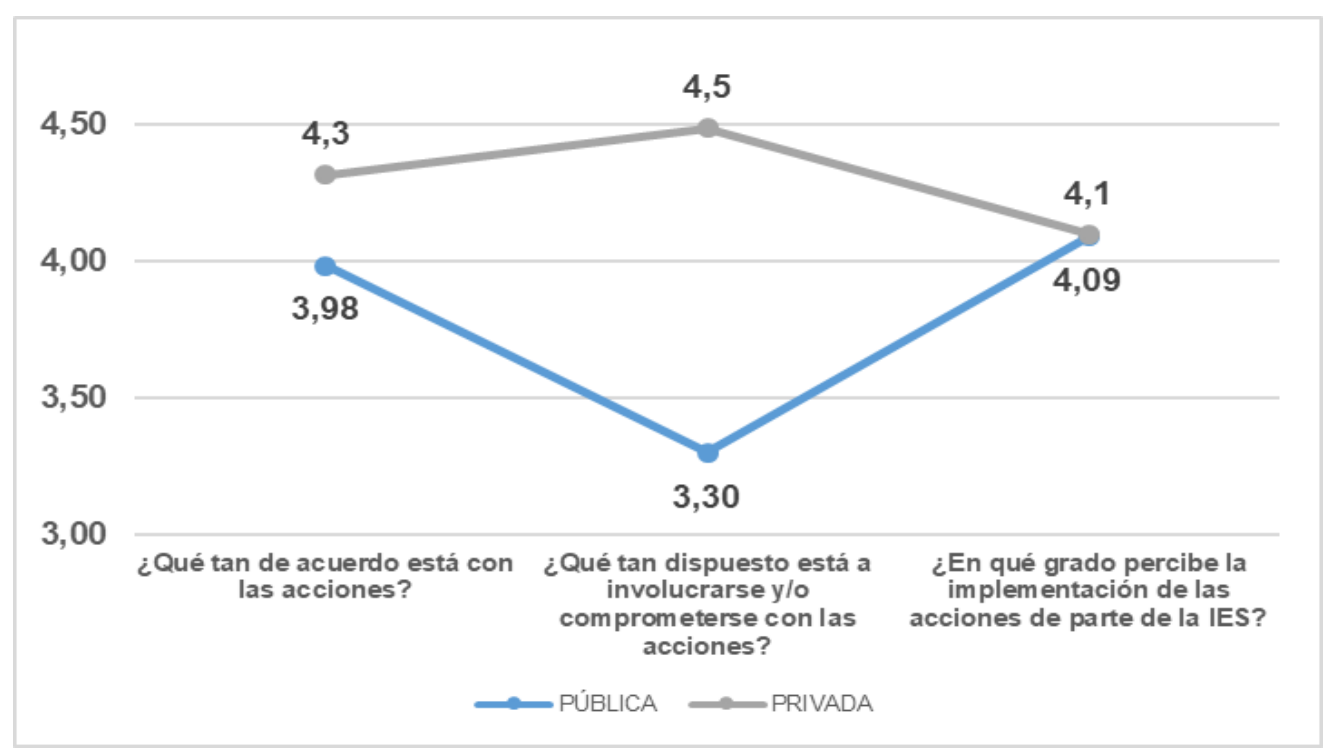

Fuente: Elaboración propia

En la tabla 5 se presentan los resultados del análisis con la prueba no paramétrica U de Mann-Whitney, para verificar si las diferencias en las variables de los niveles estudiandos son estadísticamente significativos. Se encontró de manera relevante que la Gestión Pedagógica lo es para los tres niveles así como el constructivismo. Por otro lado, la percepción de la gestión institucional, escolar y pedagógica en el nivel "grado de implementación de las acciones de parte de las IES” incluyó casi a todas las dimensiones de la gestión educativa.

Tabla 5: Relaciones estadísticamente significativas entre financiamiento público y privado $(\mathrm{p}<0.05)$

\begin{tabular}{|lll|}
\hline \multicolumn{1}{|c|}{ Nivel } & \multicolumn{1}{c|}{ Variable } & p (valor) \\
\hline \multirow{2}{*}{ ¿Qué tan de acuerdo está con } & GESTIÓN PEDAGÓGICA & 0.029 \\
las acciones? & Evaluación & 0.023 \\
& Constructivismo & 0.029 \\
\hline
\end{tabular}




\begin{tabular}{|c|c|c|}
\hline $\begin{array}{l}\text { ¿Qué tan dispuesto está a } \\
\text { involucrarse y/o comprometerse } \\
\text { con las acciones? }\end{array}$ & $\begin{array}{l}\text { GESTIÓN PEDAGÓGICA } \\
\text { Constructivismo }\end{array}$ & $\begin{array}{l}0.044 \\
0.044\end{array}$ \\
\hline $\begin{array}{l}\text { ¿En qué grado percibe la } \\
\text { implementación de las acciones } \\
\text { de parte de la IES? }\end{array}$ & $\begin{array}{l}\text { GESTIÓN INSTITUCIONAL } \\
\text { GESTIÓN ESCOLAR } \\
\text { GESTIÓN PEDAGÓGICA } \\
\text { Deserción } \\
\text { Evaluación } \\
\text { EBC } \\
\text { Impulso I+D+I } \\
\text { Vinculación } \\
\text { Extensión } \\
\text { Ética y Valores } \\
\text { Constructivismo }\end{array}$ & $\begin{array}{l}0.012 \\
0.006 \\
0.000 \\
0.023 \\
0.011 \\
0.000 \\
0.003 \\
0.001 \\
0.005 \\
0.001 \\
0.000\end{array}$ \\
\hline
\end{tabular}

Fuente: Elaboración propia

Para concluir con el análisis se aplican pruebas cuantitativas para identificar si la pertenencia a una IES pública o privada se relaciona de manera estadísticamente significativa con los tipos de gestión (dimensiones) y con los indicadores de estos. Para ello se utilizan las pruebas no paramétricas U de Mann-Whitney y Kruskall-Wallis para muestras independientes. En la Tabla 6 se muestran los resultados.

Tabla 6: Relaciones estadísticamente significativas $(p<0.05)$

\begin{tabular}{|c|c|c|c|}
\hline \multirow{2}{*}{$\begin{array}{c}\text { Acciones y categorias de } \\
\text { gestión }\end{array}$} & \multicolumn{3}{|c|}{ Variables sociodemográficas } \\
\hline & Rango de edad & $\begin{array}{c}\text { Años de experiencia } \\
\text { profesional }\end{array}$ & $\begin{array}{l}\text { IES pública o } \\
\quad \text { privada }\end{array}$ \\
\hline \multicolumn{4}{|l|}{ Acceso Universal } \\
\hline \multicolumn{4}{|l|}{ Educación de Calidad } \\
\hline Deserción & & 0.038 & 0.023 \\
\hline Evaluación & & & 0.011 \\
\hline $\mathrm{EBC}$ & & & 0.000 \\
\hline Fomento I+D+I & & 0.040 & 0.030 \\
\hline Vinculación & & & 0.010 \\
\hline Extensión & & 0.080 & 0.048 \\
\hline \multicolumn{4}{|l|}{ Uso de TIC } \\
\hline Ética y Valores & 0.037 & 0.330 & 0.001 \\
\hline Constructivismo & & & 0.029 \\
\hline
\end{tabular}


Fuente: Elaboración propia

En primer lugar vale la pena destacar que el género, el último grado de estudios, los años de experiencia docente, el nivel educativo en el que imparte y la formación pedagógica formal no aportaron evidencia de que influyan en la percepción de las dimensiones e indicadores de la gestión educativa. Así mismo, es de resaltar que el acceso universal y la educación de calidad si bien se presentan en niveles distintos, en las IES públicas y privadas, alcanzan niveles altos por lo cual no se encontró que estuvieran relacionados con variables sociodemográficas. Un hallazgo interesante es que el uso de TIC para la medicación de los procesos educativos tampoco se encuentran relacionados, esto puede dar idea de que su nivel de implementación es percibido de manera adecuada en ambos tipos de instituciones, aunque es evidente que la disposición de los docentes en las IES públicas para su implementación es claramente menor.

Cabe resaltar que la ética y valores se relacionan no sólo con el tipo de financiamiento, sino también con el rango de edad del profesor (mayor percepción entre los 36 y 55 años) y sus años de experiencia profesional (a más años mayor percepción de la ética y valores). En el caso de la deserción, fomento de la I+D+I y la extensón se observa el mismo comportamiento los más jóvenes (menores de 36) y los mayores de 55 tienen una percepción más baja que el grupo de edad que queda al centro.

Se confirma que el tipo de financiamiento de la IES (público o privado) influye en todos las dimensiones de la gestión: institucional, escolar y pedagógica, así como en la mayoría de sus indicadores. El resultado confirma lo ya mostrado, en las IES privadas los niveles de gestión educativa son mayores. 


\section{Conclusiones}

La actual gestión educativa se encuentra en una época de gran dinamismo y predomina el pensamiento estratégico de la misma implica conocer los resultados y el rendimiento, los cuales son procesos en donde se asigna o reasignan los recursos.

En cuanto a las hipótesis formuladas se encontró lo siguiente:

H1 Los niveles de gestión educativa percibida por los docentes están relacionados de manera estadísticamente significativa con el tipo de financiamiento de la IES (público o privado). Se acepta, así lo muestran los datos recolectados y las pruebas realizadas.

H2 Los niveles de gestión educativa percibida por los docentes están relacionados de manera estadísticamente significativa con su último nivel educativo. Se rechaza, no se encontró evidencia que pudiera probar esto, lo cual, pudiera ser indicativo de que la mayor habilitación no es garantía de mayor compromiso hacia las metas de las IES.

H3 Los niveles de gestión educativa percibida por los docentes están relacionados de manera estadísticamente significativa con su formación pedagógica formal. Se rechaza, al parecer la formación pedagógica formal no apoya tampoco el desarrollo de compromiso de los docentes hacia las metas de las IES.

Es de alta relevancia mencionar que los resultados del presente trabajo llevan a concluir que se distingue el rasgo principal de influencia para IES tanto privadas como públicas: la ética y valores que tiene una relación no sólo con el tipo de financiamiento sino con el rango de edad del profesor y los años de experiencia laboral. Aunque en menor medida, la deserción, el fomento a la $\mathrm{I}+\mathrm{D}+\mathrm{I}$ y la extensión también mostraron ser influenciadas por el rango de edad y el tipo de financiamiento.

La gestión pedagógica resulta ser aquella que mostró que sus diferencias de resultados (mejores en la privada que en la pública) son estadísticamente significativos, así que no sólo aplican para la muestra sino 
pueden inducirse hacia la población. Cabe destacar que las prácticas constructivistas son su indicador base.

Estos resultados complementan el paradigma sobre las innovaciones educativas contemporáneas que priorizan las prácticas de interacción comunicacional y la visión compartida y no centrada en los recursos (CASASSUS, 2002).

Ahora bien, el financiamiento como rasgo de relación marcará una diferencia entre las IES siempre y cuando se tenga un claro conocimiento de los indicadores de rendimiento, en otras palabras, en la cercanía de los resultados de su producto del modelo educativo y su referencia con estándares para a partir de eso mejorar o mantener la productividad eficiencia- y el producto educativo -eficacia-.

Descriptivamente se puede mencionar que para las IES públicas, el desafío se encuentra entre la disposición al involucramiento y el compromiso de los docentes en la acción, la cuál está evaluada con menor puntuación, especialmente para el área de vinculación. En contraparte se analiza el puntaje alto en la percepción del docente sobre grado de implementación de las acciones de las IES públicas en correspondencia con la disposiciones a realizar las acciones medidas. Mientras que para las IES privadas el desafío se concentra entre la percepción baja en grado de implementación de las acciones, especialmente para vinculación y fomento de $\mathrm{I}+\mathrm{D}+\mathrm{I}$.

Para las IES tanto privadas como públicas se vislumbran desafíos en la gestión educativa, por lo que consecuente a lo que mencionan Nava, Glasserman y Torres, (2019) existe en México una necesidad en la promoción del diálogo entre los docentes, autoridades y estudiantes sobre la calidad de los modelos y la operación institucional que identifique desafíos para facilitar una mayor disposición, compromiso en las acciones y fomento de innovación educativa.

Este trabajo es relevante porque permite establecer criterios de partida para la evaluación de la percepción de las gestión educativa y sus dimensiones en otras instituciones y contextos, así como aporta información 
acerca de la relevancia del tipo de financiamiento en cuanto a la disposición para el trabajo y compromiso de parte de los docentes. Así como nuevas líneas que identifiquen las causas y propongas estrategias para atenderlas. También se considera importante identificar si las funciones analizadas en este estudio realmente existen en las IES y si es así si realiza una plan, se asignan recursos, se implementan y se evalúan.

Las limitaciones principales de la presente investigación radican en el tamaño de la muestra y el tipo de diseño causal imposibilita la generalización de los resultados discutidos especialmente en otros contextos. Adicionalmente, sería conveniente replicar la investigación en otro contexto y observar las diferencias y similitudes que resulten de las nuevas muestras.

\section{Propuestas}

Para una mejor gestión educativa en las Universidades Mexicanas en el Siglo XXI deberán atenderse con carácter prioritario los retos siguientes ya señalados en el trabajo (Sánchez Macías y Zilberstein Toruncha, 2019), en este caso que nos ocupa resaltando los cinco retos:

1. Acceso universal y de calidad a la educación para todos los estudiantes universitarios; Reducción y atención a la deserción universitaria, con énfasis en el primer año de las carreras;

2. Centrar la educación universitaria en la preparación para el ejercicio de la profesión y el compromiso con el servicio a la sociedad;

3. Diseño de actividades que propicien el desarrollo en el contexto real de la ciencia y la tecnología;

4. Enfoque en la práctica pedagógica hacia la educación y no limitado a la instrucción o enseñanza;

5. Actuación integrada al contexto, para lo cual es imprescindible la formación y rápida inserción laboral de sus egresados, trabajar en 
redes de intercambio e involucrar a sus docentes y estudiantes en las mismas.

\section{Referencias}

ARAVENA CASTILLO, F., \& HALLINGER, P. (2018). Systematic review of research on educational leadership and management in Latin America, 1991-2017. Educational Management Administration \& Leadership, 46(2), 207-225 DOI: $10.1177 / 1741143217745882$.

BALDERRAMA, S. (2006). Al Grano con el Plan estratégico de Transformación escolar. Impresora Gobierno del Estado de Chihuahua. Segunda Reimpresión. México.

BARBÓN PÉREZ, O. G. Y FERNÁNDEZ PINO, J. W. (2018). Rol de la gestión educativa estratégica en la gestióndel conocimiento, la ciencia, la tecnología y lainnovación en la educación superior. Educación Médica, 19(1), 51-55 Disponible en https://doi.org/10.1016/j.edumed.2016.12.001.

BERTEL-NARVÁEZ, M. P., VILORIA-ESCOBAR, J. D., \& SÁNCHEZ-BUITRAGO, J. O. (2019). Tendencias de investigación en los posgrados de gestión educativa en América Latina. Educación y Educadores, 22(2), 215-233. DOI:

http://dx.doi.org/10.5294/edu.2019.22.2.3.

CASASSUS, J. (2002). Problemas de la gestión educativa en América Latina: la tensión entre los paradigmas de tipo A y el tipo B. Santiago de Chile: Unesco.

CONNOLLY, M., JAMES, C., \& FERTIG, M. (2019). The difference between educational management and educational leadership and the importance of educational responsibility. Educational Management Administration \& Leadership, 47(4), 504-519 DOI: 10.1177/1741143217745880.

DI BELLO, M. Y ROMERO, L. A. (2018). Vinculación y extensión universitaria: la relación entre la universidad y sus entornos en las universidades nacionales de Quilmes y Lanús. Apuntes. Revista De Ciencias Sociales, 45(82), 145-171. doi:https://doi.org/10.21678/apuntes.82.867.

FAYOL, H. (1949). General and industrial management. New York: Martino Publishing.

FRANCO GARCÍA, M. J. (2018). La gestión educativa en México: una política sin consenso de la base. Temas De Educación, 23(1), 85-110. Recuperado de https://revistas.userena.cl/index.php/teduacion/article/view/1004.

GARCÍA GALVÁN, R. (2017). Fomento a la ciencia y a la tecnología en las universidades de México. Ciencia UANL, 20(83). Recuperado de http://cienciauanl.uanl.mx/?p=6884. 
GLASSERMAN MORALES, L. D., TORRES ARCADIA, C. C., \& NAVA LARA, S. A. (2019). Innovación educativa en estudios sobre gestión educativa: una revisión sistemática de literatura. España: Octaedro. Recuperado de: http://repositorio.grial.eu/handle/grial/1821.

HERNÁNDEZ ROJAS, G. (1998). Paradigmas en Psicología de la Educación. México: Paidós.

LEMOS, M. (2017). Collaborative agency in educational management: a joint object for school and community transformation. Revista de Administração de Empresas, 57(6), 555-566. Recuperado de: https://doi.org/10.1590/s0034-759020170604.

MEDINA PALOMERA, A., AMADO MORENO, M. G. Y BRITO PÁEZ, R. A. (2010). Competencias genéricas en la Educación Superior Tecnológica mexicana:. Actualidades Investigativas en Educación, 10(3), 1-28. Recuperado de https://www.redalyc.org/articulo.oa?id=44717980008.

OPLATKA, I. (2019). El surgimiento de la gestión educativa como campo de estudio en América Latina. Reveduc, 13(1), 196-210 DOI:

http://dx.doi.org/10.14244/198271993072.

PIGOZZI, M. J. (2008). Towards an index of quality education. París: UNESCO.

Recuperado el 26 de 02 de 2019, de

http://www.ibe.unesco.org/fileadmin/user upload/COPs/Pages documents/Resource _Packs/TTCD/sitemap/resources/1_1_2_P SPA.pdf.

SÁNCHEZ MACÍAS, A. (2013). ¿Administración o Gestión educativa? Un ejercicio conceptual. Antología Revista Conexxión. Año 4. 2012-2015. México. 1-11.

Recuperado de: http://www.aliatuniversidades.com.mx/conexxion/wpcontent/uploads/2016/pdf/esp2.pdf.

SÁNCHEZ MACÍAS, A. (2016). Dimensión económica de la educación: eficacia y eficiencia. Educando para Educar,, 16(30), 0-38. Recuperado de:

https:/www.researchgate.net/publication/330093034 Dimension economica de la educacion eficacia y eficiencia.

SÁNCHEZ MACÍAS, A. Y VEYTIA BUCHELI, M. G. (2019). Digital competences in doctoral students. A study at two Mexican universities. Academia y virtualidad, 1(12), 1-29. DOI: https://doi.org/10.18359/ravi.3618.

SÁNCHEZ MACÍAS, A. Y ZILBERSTEIN TORUNCHA, J. (2019). Retos a la Educación Superior en las primeras dos décadas del siglo XXI: ¿qué ofrecer a la sociedad? Memorias de III Encuentro Bilateral Cuba-México. Ciencias de la Educación y Ciencias Administrativas (págs. 1-16). Varadero, Cuba: Universidad de Matanzas.

SEMS. (2014). Movimiento contra el Abandono Escolar en la Educación Media Superior. Principales estrategias internacionales para reducir la deserción escolar. Obtenido de Subsecretaría de Educación Media Superior. Secretaría de Educación 
Pública, México. Recuperado de:

http://www.sems.gob.mx/work/models/sems/Resource/11390/1/images/000_INTROD UCCION_Movimiento_contra_Abandono.pdf.

SEP. (2009). Modelo de Gestión Educativa Estratégica. Programa Escuelas de Calidad. México: Secretaría de Educación Pública. Recuperado de: Universidad de Pamplona. (2015). Eje transversal axiológico. Recuperado dehttp://www.unipamplona.edu.co/unipamplona/portalIG/home_9/recursos/general/ 12062015/eje_transversal_axiologico.pdf.

VALERA SIERRA, R. (2010). El proceso de formación del profesional en la educación superior basado en competencias: el desafío de su calidad, en busca de una mayor integralidad de los egresados. Civilizar. Ciencias Sociales y Humanas, 10(18), 117-134. Recuperado de https://www.redalyc.org/articulo.oa?id=100220339010.

ZILBERSTEIN, J., (2015). Reformas y transformaciones de la Educación Básica actual: Hacia una Educación Basada en Competencias. Sus retos para la gestión escolar. En: Antología Revista Conexxión. Año 4. 2012-2015. México. 125-142. Recuperado de: http://www.aliatuniversidades.com.mx/conexxion/wpcontent/uploads/2016/pdf/esp2.pdf.

ZILBERSTEIN, J., SILVESTRE, M., OLMEDO, S., (2015). Diagnóstico y transformación de la institución docente. Ediciones CEIDE. México. 